\title{
XXXIV. On the mechanics of luminosity
}

\section{E. Wiedemann}

To cite this article: E. Wiedemann (1889) XXXIV. On the mechanics of luminosity, Philosophical Magazine Series 5, 28:173, 248-267, DOI: 10.1080/14786448908621354

To link to this article: http://dx.doi.org/10.1080/14786448908621354

\section{Published online: 08 May 2009.}

Submit your article to this journal 준

LII Article views: 3

Q View related articles $\asymp$ 
former, being the difference of two curvatures, is itself a curvature. If the curvature thus determined by shape solely is expressed in dioptries, then, on multiplying by the numeric which depends on the nature of the material, the resultant power of the lens will also be expressed directly in dioptries. The dioptrie spherometer facilitates this desirable end by its directness of reading, quite apart from any incidental advantages which may result from the details of construction.

XXXIV. On the Mechanics of Luminosity.

By E. Wiedemann.

[Continued from p. 163.]

Experimental Measurements.

17. T $\mathrm{N}$ order to show how many of the questions raised in 1 the foregoing admit of an experimental solution I have made a number of measurements in various directions. The method of investigation is briefly the following :-

Since in all measurements the coefficients of emission are to be expressed in calorimetric measure, $i . e$. in gramme-calories per second, the numbers given by the amyl-acetate lamp, serving as the source of light, for comparison at a given distance from the photometer-slit must be reduced to absolute intensity. This was done by comparison with the radiation of a glowing platinum wire. From these measurements we obtain at once the energy in gramme-calories per second radiated by $1 \mathrm{gr}$. platinum, and, consequently, relationships between the total energy and that radiated in a definite region of the spectrum, e.g. in the yellow. Next, the total brightness of gas-flame coloured with sodium was compared with the brightness of the amyl-acetate lamp in the yellow ; and hence the coefficient of emission was calculated in grammecalories per second for one gramme and for a molecule of sodium.

Then follows the determination of the quantity $b$, and from its value that of the store of luminous energy $L$, and therefore of the kinetic energy of the luminous motions.

\section{Dependence of the Radiation of a Platinum Wire upon its Temperature.}

18. The measurements were carried out in the first place for the D-line. The amyl-acetate lamp was always employed as the comparison-lamp.

The arrangement followed in these measurements and for 
the determination of the brightness of the comparison-lamp in absolute measure was the following:-At a definite distanco from the slit in the prolongation of the axis of the collimator of a Glan's photometer a platinum wire was placed, with its axis of length parallel to the slit, which was then ignited by means of a galvanic current. The source of light was placed before the comparison-prism.

The platinum wire $d$ must be placed in a vacuum, since otherwise its loss of energy would depend not only-nor even chiefly-upon the radiation, but very largely also upon convection-currents. Since the wire expands when heated, it must not be clamped tight or it will become curved, and would thus not remain parallel to the slit. It was therefore fastened in the following manner (Pl. III. fig. 1). Its upper end was soldered to a strong brass wire $m$, at its lower end a long thick copper rod $k$ was fastened, provided below with a point $p$. Above and below, the outer portions of two ground pieces $\sigma$ and $\sigma^{\prime}$ were melted on to a cylindrical glass tube $c$ of 38 millim. diameter. A brass wire $m$ was cemented into the inner portion $s$ belonging to the upper ground portion. The inner portion $s^{\prime}$ of the lower ground portion contained mercury $q$, which was put into connexion with the source of electricity by means of the copper wire $r$ cemented in, into which the point $p$ of the copper rod $k$ plunged. When the platinum wire was heated by means of a current, the copper wire was stretched by the copper rod so that it remained perfectly straight. A side tube $h$ connected the cylindrical tube with Töpler's mercury pump with Sundell's modifications and improvements*. It was exhausted as completely as possible. In a discharge-tube melted on at the same time and provided with a plate and a point 6 centim. from each other, discharges were scarcely to be obtained, using an induction-coil of moderate size. In fact the further action of the pump did not diminish the brightness of the wire. The heating of the glass wall by radiation was without influence, since, as far as the measurements of brightness and the electrical measurements were concerned, it was a matter of indifference whether the wire was maintained at the high temperature a longer or a shorter time.

The double-image prism in the photometer was so placed that the squares of the cotangents of the angles read were proportional to the brightness $\mathbf{J}$ of the platinum wire. The apparatus furnished to me differed from that described by Herr Glan in having the Wollaston's prism turned through an angle of $180^{\circ}$.

* Beibl. ix. p. 756 (1885). 
The quantity of energy $\mathrm{E}$ lost per second by radiation during the constant luminosity of the platinum wire is proportional to the product of the resistance $w$ of the wire, and the square of the intensity of the current $i$, or, if they are measured in ohms and amperes,

$$
\mathrm{E}=0.24 w i^{2} \text {, }
$$

where 0.24 is the necessary factor of reduction to convert energies measured in ohms and amperes into gramme-calories per second*.

In order to determine these quantities a Wheatstone's bridge (fig. 2) was constructed in the following mannert:-

The branch 1 contained a vertical rectangular bar of wood, $\alpha$, surrounded by oil, upon which various constant known resistances were formed, as well as the reflecting galvanometer $g_{1}$, serving to measure the strength of the current with the strong currents which had to be used here. A thick copper ring was employed instead of a multiplier, the resistance of which and its connexions was $\kappa$.

The branch 2 was formed by the platinum wire $d$ to be investigated, whose resistance at temperature $t$ may be called $w_{t}$.

The branch 3 contained a large resistance of $y$ nominal Siemens units, and branch 4 a box of resistance-coils of from 1 to 5000 Siemens units, the accuracy of which had been previously tested.

The heating-current entered at the point 1,3 , and was furnished by six large Bunsen cells arranged three in series (the reason for not employing a Gramme or dynamo is given further on), and was led off at the point 2,4 . A variable resistance included in the circuit at $W$ permitted the variation of the strength of the current, and with it the heat produced, and consequently the luminosity.

The resistance of the branches 3 and 4 was always so great in comparison with 1 and 2 that the variations of the current caused no perceptible variation in the intensity of the current in 1 and 2 . (The resistance of 1 and 2 never exceeded 2 Siemens units, and the resistance in 3 and 4 was not less than 400 Siemens units.)

The sensitive galvanometer $g_{2}$ serving for the measurement of the resistances was inserted between the points 1,2 and 3,4 . By changing the plugs in the box of resistance-coils in branch 4 the resistance was altered until the galvanometer remained at rest on opening and closing the key.

* Kohlrausch, Leitfaden d. pract. Physik. 6th ed. p. 334 (1887). (1887).

+ Compare Bottomley, Phil. Trans. Roy. Soc. London, exviii. p. 499 
If the resistances in $1,2,3,4$ are $w_{1}, w_{2}, w_{3}, w_{4}$, then $w_{1}: w_{2}=w_{3}: w_{4}$. But since $w_{1}, w_{3}, w_{4}$ are known, we have at once $w_{2}$, the resistance of the platinum wire under investigation. After the conclusion of each series of experiments the constant of the galvanometer $g_{1}$ was determined by means of a voltameter.

The resistance of the platinum wire at the ordinary temperature of $15^{\circ}$ was determined by means of an ordinary Wheatstone bridge, with one Leclanché cell to furnish the current, so as to avoid heating. The resistances $a, m$, and $g$ were found in the same way.

19. The first series of experiments had for its object to determine how the intensity of light in the yellow increased as the total radiation increased upon rise of temperature. The region of the spectrum examined was close to the sodium line. The platinum wire was at a distance of 45 millim. from the slit. The amyl-acetate lamp was at a distance of 20 centim. Then for various degrees of luminosity we obtain the resistances $w_{t}$, their ratio to the resistance $w_{10}$ at $15^{\circ} \mathrm{C}, \frac{w}{w_{15}}$, the intensities of the current $i$ and the quantities $w_{i} i^{2}$ proportional to the total radiation (assuming that the wire has the same temperature at all points of its section), and, lastly, the brightness $J$ in the yellow.

$$
\begin{aligned}
& w_{10}=0 \cdot 2555 \\
& \begin{array}{cllll}
w_{t}=0.6314 & 06609 & 0.6923 & 0.7274 & 0.7736 \\
i=1.997 & 2 \cdot 195 & 2 \cdot 478 & 2.843 & 3.383
\end{array} \\
& \frac{w_{t}}{w_{15}}=2 \cdot 471 \quad 2.587 \quad 2.709 \quad 2.847 \quad 3.028 \\
& w_{i} i^{2}=2.518 \quad 3.184 \quad 4.251 \quad 5.879 \quad 8.854 \\
& \mathrm{~J}=0.025 \quad 0.0935 \quad 0.189 \quad 0.455 \quad 1.761
\end{aligned}
$$

According to the data of Sir William Siemens", the ratio $w_{t} / w_{15}=2.471$ would correspond to about $700^{\circ} \mathrm{C}$., and that of 3028 to about $1000^{\circ} \mathrm{C}$.; with which the observation agrees that in my experiments the brightness increased seventy times; whilst, according to M. Violle $\dagger$, it increased for the portion of the spectrum corresponding to the D-line from 0.05 to $3 \cdot 6$ between $715^{\circ} \mathrm{C}$. and $1045^{\circ} \mathrm{C}$., that is, about seventy-two times.

The above values show that the radiation in the visible spectrum rises much more rapidly than the total radiation. The former $(J)$ increases 70 times, the latter $(E$, measured by means of $\left.w i^{2}\right) 3 \frac{1}{2}$ times.

* Proc. Roy. Soc. Lond. xxxv. p. 166 (1883).

$\dagger$ Comptes Rendus, xcii. pp. 866 \& 1204 (1881). 
Within the visible spectrum the like is shown by older experiments. Thus, M. Violle finds that between $954^{\circ}$ and $1775^{\circ}$ the intensity for the C-line increases 154 times, for the D-line 219 times, and for the E-line 307 times. The intensity therefore increases the more slowly the less refrangible the rays are. Moreover the total radiation contains the infrared rays, whose increase will be much slower than is shown by the above numbers. At the same time we see that even at the highest temperature the radiation in the infra-red furnishes an overwhelming contribution to the total radiation. For the extraordinarily rapid increase of the radiation in the visible part of the spectrum is not sufficient, as the numbers given above show, to compensate the very much slower increase in the infra-red.

Herr H. Schneebeli * has obtained the same results in experiments with Swan lamps. Whilst the total radiation increased in the proportion of 1 to 4 , the optical radiation increased in the proportion of 0.3 to 24 . The Swan lamps are, however, scarcely so highly exhausted but that one must suppose that a portion of the loss of energy is due to convection. Further H. Schneebeli employed a gramme-machine to furnish the current, of which the current-strength varied between certain maximum and minimum values. The quantity of energy given off in the unit time is then, if we denote by $\mathrm{J}$ the mean current-strength as shown by the galvanometer, and by $i$ that existing at any time $t$, and by $w$ the resistance regarded as constant, not $u \mathrm{~J}^{2}$, but $\int_{0}^{1} w i^{2} d t$. It is not possible to decide between these two values without knowing what the action of the Gramme-machine was.

When $\mathrm{Hr}$. E. Lecher $\uparrow$ nevertheless, in a research on the visible spectrum, is able to make the brightness equal in all parts of the spectra yielded by two different platinum wires by weakening the whole of the brighter of the two spectra, the reason no doubt is that, as the above-mentioned experiments of M. Violle show, the changes in the visible spectrum for the different colours are not remarkably different. Moreover, for the small brightnesses in question, only quite limited regions of the spectrum contribute to the physiological lightsensation $\ddagger$.

* Wied. Ann. xxii. p. 433 (1882). † Ibid. xvii. p. 512 (1882).

$\ddagger$ I wish to avail myself of this opportunity to make a remark on the construction of formulæ which represent the emission as a function of the temperature and the wave-length. With the exception of the formula of Herr Stefan, according to which the radiation is proportional to the fourth power of the absolute temperature, all have more or less the character of interpolation-formulæ.

If a formula, based upon theoretical considerations, is really to represent 


\section{Total Radiation from one Square Centimetre and one Gramme Platinum.}

20. In the determination made for the purpose of evaluating the amyl-acetate lamp in absolute measure, the distance between the slit and the wire was 44 millim. This is made up of the distance of the slit from the edge of the glass tube and the radius of the tube. The distance of the amyl-acetate lamp from the slit was 216 millim. The length of the wire was $7 \cdot 6$, its thickness $0 \cdot 26$ millim. Consequently the radiating surface $\mathrm{O}=2 \pi r l=0.6205$ square centim.

If the current-strength (in amperes) is $i$, the resistance for current-strength equal to $w_{1}$ (in ohms $\Omega$ ), the resistance at $15^{\circ} w_{15}$, the energy radiated by the whole wire $\mathrm{F}=0 \cdot 24 w i^{2}$ (in $\mathrm{cm}$. g. sec. cal.), the readings $\alpha$ on the photometer for the part of the spectrum close to the sodium-line, the brightness J measured by $\operatorname{cotan}^{2} \alpha$, the energy radiated by the unit surface $\mathrm{E}=\mathrm{G} \div 0$, then the following (amongst other) data were obtained :-

$$
\begin{array}{rlrl}
w_{15} & =0.239 \Omega, & w_{i} & =0.757 \Omega, \quad w_{i} / w_{15}=3 \cdot 17 ; \\
i & =4.00, \quad \mathrm{~F} & =2.91, \quad \mathrm{E}=4.68 ; \\
\alpha & =36^{\circ} 30^{\prime}, & \mathrm{J} & =\operatorname{cotan}^{2} \alpha=1.827 .
\end{array}
$$

A square centimetre of the surface of a platinum wire heated to about $1000^{\circ} \mathrm{C}$. consequently radiates about $4 \cdot 7 \mathrm{gr}$. calories in a second.

The value of $\mathrm{E}$ agrees in order of magnitude with that of H. Bottomley* for the highest temperature used by him; mine is higher. This is explained by the fact that the temperature used by me is higher than his $\dagger$.

the above-mentioned dependence over a considerable region, then in the first place no luminescence-phenomena must occur with the body in question, and in the second place the molecules and atoms which with their æther envelopes build up the body must undergo no changes. But such a change certainly takes place in all bodies which show a great change in specific heat upon increase of temperature. Sivee the specific heats in the liquid and gaseous and in the liquid and solid conditions exhibit like coefficients of change, so far at least as present investigations extend, the quantity of heat corresponding to the change in specific heat can only result from intramolecular work. But anything that brings about an increased porosity of the molecular condition, or an increased intramolecular activity, must modify the emission and absorption of light in a way not immediately determinable.

* Phil. Trans. cxviii. p. 429 (1887).

+ It is only after completion of the present incestigation that I have become acquainted with the work of Messrs. O. Tumlirz and A. Krug, "On the Energy of the Radiation of Heat at a White Heat" (Sitzungsber. d. Wien. Akad. xcvii. pp. 1521-1529, 13/12, 1880), which follows a provious paper by one of the authors (Wien. Ber. xcvi. p. 1007, 1888); as Phil. Mag. S. 5. Vol. 28. No. 173. Oct. 1889. 
The number 4.7 obtained for the total energy is taken as the basis of all the following calculations.

21. From these numbers certain further conclusions can be obtained.

We will first calculate the quantity of energy radiated per second from 1 gramme and from one atom of platinum.

The above quantity of energy, $4 \cdot 7$ calories, is emitted by a layer of unit surface, which has such a thickness that the rays coming from the deepest points still perceptibly issue from the surface. The different layers contribute in different degrees to the radiation. For the sake of simplicity we will assume that all the layers do this equally. If $d$ is the thickness of the radiating layer, $s$ the specific gravity of the radiating substance, then the weight of the radiating layer under the unit surface is $\gamma=s d$. For $d$ I put the approximate value $d=10^{-5}$ $\mathrm{cm}$., corresponding to the numbers of Herr Wien *. Further, for platinum, $s=21.5$; then the weight of the radiating layer for platinum is $2.15 \times 10^{-4} \mathrm{gr}$. One gramme of platinum consequently emits in one second a quantity of energy, $2 \cdot 2 \times 10^{4}$.

The total emission-coefficient of 1 gramme of platinum at the temperature used of about $1000^{\circ} \mathrm{C}$. is therefore

$$
\mathrm{S}=2 \cdot 2 \times 10^{4} \mathrm{gr} \text {. calories per second. }
$$

But further, according to the calculations of van der Waals $\dagger$, the absolute weight of a single atom of hydrogen is $7 \cdot 5 \times 10^{-23} \mathrm{gr}$., the atomic weight of platinum 194 ; consequently the weight of an atom of platinum is, in round numbers, $15 \times 10^{-21} \mathrm{gr}$. Consequently an atom of platinum at the temperature of about $1000^{\circ} \mathrm{C}$., assumed for the purposes of our calculations as that of the platinum wire, emits $2 \cdot 2 \times 10^{4} \times$ $15 \times 10^{-21}=3.3 \times 10^{-16} \mathrm{gr}$. calories per second.

We will further determine the relationship existing between the quantity of heat emitted at $1000^{\circ} \mathrm{C}$. and the quantity

well as that of Herr Tumlirz, "Calculation of the Mechanical Equivalent of Light from the experiments of H. J. Thomsen" (Ibid. pp. 1625-1632. 20,12. 1888). This gives the radiation of glowing platinum per square centimetre, and a comparison of the brightness of the glowing platinum with the acetate lamp. My values agree with his also as to order of magnitude. The differences may be explained by the fact that different wires behave differently; and that these gentlemen, whose object was the exact determination of this magnitude, have introduced a number of corrections which I did not consider necessary. As I was only concerned to determine the order of magnitude of the several quantities, nothing essential in the conclusions is altered by the introduction of the values of other observers instead of mine.

* Wied. Ann. xxxv. p. 57 (1888).

† Cf. R. Ruhlmann, Mech. Wärmetheorie, ii. p. 247 (1885). 
required to raise the quantity of platinum in question from $0^{\circ}$ C. to $1000^{\circ}$.

The mean specific heat of platinum between $0^{\circ}$ and $t^{\circ}$, according to Violle*, is

$$
c_{0}^{t}=0.0317+0.0_{5} 6 t ;
$$

and therefore between $0^{\circ}$ and $1000^{\circ} \mathrm{C}$.,

$$
c_{0}^{1000}=0.038 \text {. }
$$

To heat one gramme from $0^{\circ} \mathrm{C}$. to $1000^{\circ} \mathrm{C}$. we therefore require $1000 \times 0.038=38$ calories; and to heat the surfacelayer,

$$
2 \times 10^{-4} \times 38 \mathrm{cal} .=7 \cdot 6 \times 10^{-3} \mathrm{cal} .
$$

The quantity of energy radiated per second at $1000^{\circ}$ is therefore about 600 times greater than the quantity communicated in heating from $0^{\circ}$ to $1000^{\circ}$.

If, further, we have a platinum wire of $r$ centim. radius and 1 centim. length, then at $1000^{\circ}$ the quantity of energy lost per second by radiation $\mathrm{M}$, and the quantity $\mathrm{W}$ communicated in heating from $0^{\circ}$ to $1000^{\circ}$, are given by the formula therefore

$$
\begin{gathered}
\mathrm{M}=2 \pi r \times 4.7, \quad \mathrm{~W}=\pi r^{2} \times 21.5 \times 38 ; \\
\mathrm{W} / \mathrm{M}=87 \times r .
\end{gathered}
$$

We see from this that, with a wire about $\frac{1}{42}$ centim. in thickness, the energy radiated in a second and that communicated in heating from $0^{\circ}$ are nearly equal. With thinner wires the latter diminishes very rapidly in comparison with the former.

Exactly similar considerations of course apply to the case of glowing and radiating platinum foil.

22. The method employed for the determination of the radiation gives it, in the first place, according to order of magnitude. The numbers just quoted show, in fact, how extraordinarily great the radiation is. The surface-layer must therefore cool rapidly. The loss of energy thus caused is instantly supplied by conduction from the interior hot portions at the expense of the work done by the current. Since the outer portions are, in any case, cooler than the inner, their resistance must be less. But the resistance measured is a mean of the variots concentric layers. Therefore it is not at once possible, without a thorough inquiry into the relationships of conductivity for heat \&c., to obtain a reliable conclusion, from the observed resistances, as to the actual tempera. ture of the radiating surface $\uparrow$.

* Comptes Rendus, lxxxv. p. 543 (1877).

$\dagger$ Compare also (amongst others) the work of G. Basso, Natura, iii. pp. 225,304 . 
The influence of these disturbing circumstances may be determined by heating a platinum wire to a definite temperature in an air-bath and measuring the intensity of the light emitted at a definite part of the spectrum, and at the same time determining its resistance. Then the wire is heated by means of a current to the same brightness and its resistance determined again. From the difference in resistance observed in the two cases account can be taken of the complications in question. The experiments should be made with wires of different thickness.

\section{Comparison of the Amyl-acetate Lamp with Glowing Platinum.}

23. From these determinations we will turn to the definite evaluation of the amyl-acetate lamp in absolute measure. Care must be taken that the platinum wire is linear, but the amyl-acetate flame, on the other hand, flat; i. e. so that the rays from the former traversing the slit only fill a portion of the objective, whilst those from the latterly entirely fill it so soon as the flame is sufficiently near to the slit, as is the case in our experiments.

How account is to be taken of these circumstances in their influence on the brightness is explained in the following.

(a) First, we calculate the quantity of energy reaching unit length of the slit from the platinum wire.

Let $h$ be the diameter of the diaphragm in the collimator which limits the pencil of rays issuing from it, $e$ its distance from the slit, $\eta$ the distance of the wire from the slit, and $\delta$ the thickness of the wire. The pencil of rays drawn from the diaphragm through a point of the slit intersects on the surface of the wire an area which, projected on the meridian-plane at right angles to the axis of the collimator, has a breadth $\delta$ and a height $\gamma$, as calculated from the proportion

$$
\gamma: \eta=h: e, \quad \gamma=\frac{\eta h}{e} .
$$

The quantity $h / e$ occurring here can be calculated as follows. At a distance $a$ of 35 centim. from the slit a scale was placed at right angles to the axis of the collimator and to the length of the slit, and a light was moved along the scale until an observer at the telescope announced that the light could no longer be seen. On moving the eye sideways right or left the distance of these two points was $2 \cdot 2$ centim. : then

$$
h / e=l / a=\frac{2 \cdot 2}{35 \cdot 0}=0 \cdot 063 \text {. }
$$


Let us take, as a first approximation, the law of cosines * as holding good for the radiation, then we replace the semicylindric surface of the wire radiating to each point of the slit by the rectangle $\gamma \delta=f$.

Let the quantity of energy radiated by each square centimetre be $\mathrm{E}$, then the surface $f$ gives, on the whole, the quantity of energy $\mathrm{E} f$.

If the width of the slit is $s$, then the unit of length of the slit receives a portion which is to the total radiation as the surface of the slit corresponding to the unit of length $s .1$ is to the half surface of a sphere of radius $\eta$. (In the quantity $\mathbf{E}$ determined by experiment we have only the quantity of energy radiated outwards, and not that radiated towards the interior of the wire.) This fraction is $s / 2 \pi \eta^{2}$.

Hence upon the unit length of the slit there is radiated from the surface $f$ of the glowing platinum wire a quantity of energy

$$
\mathrm{A}=\frac{s}{2 \pi \eta^{2}} \mathrm{E} f=\frac{\eta h}{e} \delta \frac{s}{2 \pi \eta^{2}} \mathrm{E}=\left(\frac{h}{e}\right) \frac{\delta \cdot s}{2 \pi \eta} \mathrm{E} .
$$

In our experiments

consequently

$$
h / e=0.063, \quad \delta=0.026 \mathrm{~cm} ., \quad \eta=4.4 \mathrm{~cm} \text {; }
$$

$$
\mathrm{A}=0.063 \frac{0.026^{\circ}}{2 \pi \times 4.4} \mathrm{~s} \mathrm{E}=0 \cdot 0_{4} 6 s \mathrm{E} \text {. }
$$

(b) We will now calculate the similar expression for the energy sent to the slit by a flat-shaped source of light of considerable extent, like that of the amyl-acetate lamp, or the Bunsen flame coloured with sodium, which is so near to the slit that the cone passing through the diaphragm of the collimator and a point of the slit in its prolongation towards the flame is completely filled with luminous particles.

The cone from the diaphragm through a point of the slit cuts the flame in a circle ; if the flame is at a distance $\eta^{\prime}$ from the slit, and if $\delta^{\prime}$ is the diameter of this circle, then the

* The validity of the law of cosines may, upon theoretical grounds, be open to doubt. As is well known, it is established by regarding as the radiating quantity that contained in a parallelopiped whose base is the radiating surface, and of which the edge forms a portion of the prolongation of the rays under investigation, equal to the depth from which, in general, rays still issue. But it is certainly not the particles contained in this parallelopiped which give the rays issuing in the direction in question, since in their introduction the refraction from metal into air is neglected, of the existence of which (even before the direct proof given by IHerr A. Kundt) evidence was offered by the strong polarization of the emergent light. Further experimental investigations are required to explain the contradiction between theory and the observations of Herr Möller (Wied. Ann. xxvi. p. 266), which tend to confirm the law of the cosine. 
radiating surface is

$$
\frac{\pi}{4} \delta^{2}=\frac{\pi}{4}\left(\frac{h}{e}\right)^{2} \eta^{\prime 2}
$$

If a square centimetre emits a quantity of energy $E^{\prime}$, then our surface yields a quantity of energy,

$$
\frac{\pi}{4}\left(\frac{h}{e}\right)^{2} \eta^{\prime 2} \mathbf{E}^{\prime}
$$

Of this the fraction which reaches the unit length of the slit is $8 / 4 \pi \eta^{\prime 2}$. We must here divide by the whole surface of the cone, since the sodium flame is transparent to its own rays. The quantity of energy actually falling upon the slit is therefore

$$
\mathrm{A}^{\prime}=\frac{s}{4 \pi \eta^{22}} \cdot \frac{\pi}{4}\left(\frac{h}{e}\right)^{2} \eta^{\prime 2} \mathrm{E}^{\prime}=\frac{s}{16}\left(\frac{h}{e}\right)^{2} \mathrm{E}^{\prime}=0.00_{3} 25 \& \mathrm{E}^{\prime} .
$$

The distance $\eta^{\prime}$ thus does not occur in the final result, since the radiating surfaces increase as the squares of the distances. We may say that the quantity $\mathrm{A}^{\prime}$ is the fraction of the total energy which passes through the diaphragm. Strictly speaking, account should also be taken of the circumstance that the flame represents not a space bounded by two parallel very large surfaces, but a cylinder. Nevertheless, what we thus neglect is small in comparison with the other sources of error. We have further neglected the fact that the slit is not a portion of the sphere, but occupies a tangent plane.

(c) We therefore obtain for the ratio of the energies which reach the slit from an extended source of light, and a narrow linear source

$$
\frac{\mathrm{A}^{\prime}}{\overline{\mathrm{A}}}=\frac{1}{16}\left(\frac{h}{e}\right) \frac{2 \pi \eta}{\delta} \overline{\mathrm{E}^{\prime}} \text {, or } \frac{\mathrm{E}^{\prime}}{\mathrm{E}}=16\left(\frac{e}{h}\right) \frac{\delta}{2 \pi \eta} \frac{\mathrm{A}^{\prime}}{\mathrm{A}} .
$$

With the dimensions of our apparatus in particular

$$
\frac{\mathrm{E}^{\prime}}{\mathrm{E}}=0 \cdot 24 \frac{\mathrm{A}^{\prime}}{\mathrm{A}}, \text { or } \mathrm{E}^{\prime}=0 \cdot 24 \frac{\mathrm{A}^{\prime}}{\mathrm{A}} \mathrm{E} .
$$

The ratio of the energy of a source of light with a continuous spectrum, and that of the platinum wire at a definite point of the spectrum is obtained at once from the readings of the photometer. We have seen above that the brightness of the platinum is 1.827 times greater than that of the amylacetate lamp for the yellow in the neighbourhood of the $D$ line. Hence

$$
\begin{aligned}
& A / A^{\prime}=1.827, \\
& A^{\prime} / A=0.547,
\end{aligned}
$$

and we obtain for the energy of unit surface of the amyl- 
acetate lamp expressed in terms of that of the glowing platinum for the yellow

$$
\mathrm{E}^{\prime}=0.24 .0 .547 \mathrm{E}=0.13 \mathrm{E} \text {. }
$$

\section{Comparison of Sodium Flame and Glowing Platinun.}

24. After this determination we may further compare the brightness of the amyl acetate lamp for the yellow with that of a gas-flame coloured yellow by sodium, according to the method of Herr Ebert*, and thus the latter also with the brightness of the yellow of glowing platinum.

If, then, we wish to determine the ratio of the radiation of the sodium flame corresponding only to the yellow sodium lines and the total energy of radiation of the glowing platinum, we must first determine the ratio of the latter to the radiation which reaches a definite portion of the yellow.

For this purpose we will make use of the results of Mouton $\dagger$, by assuming, without doubt correctly, that the temperature of the platinum wire in our absolute measurements is nearly equal to that of the platinum wire in Mouton's Bourbouze lamp.

If this is not exactly the case, and consequently the final value is not quite accurate, yet its order of magnitude can in no case be affected.

In order to obtain a part of the radiated energy which belongs to a definite portion of the spectrum situated in the neighbourhood of the D-line, the following method was adopted. A curve was drawn upon paper according to Mouton's numbers, which represented the distribution of energy as a function of the wave-length. The wave-lengths were measured in $1 \mu$, the energies in any convenient unit By division of the weight $g$ of a piece of the curve-paper of known area by the weight $G$ of the area included between the curve and the axis of abscissæ, we obtain for the fraction of the total energy corresponding to unit area

$$
a=\frac{g}{\mathrm{G}} \text {. }
$$

-Wied. Ann. xxxii. p. 345 (1887).

† Compt. Rend. lxxxix. p. 295,1879 ; Beibl. iii. p. 868, 1879. The following calculation of course proceeds upon the assumption that we obtain the whole quantity of radiated energy in the bolometer or the thermopile, or that the substance of the bolometer absorbs even the extreme infru-red rays. This may be tested experimentally by comparing the curvo of energy determined by the bolometer with the total expenditure of energy as measured by resistance and intensity. I should bave liked to have determined the distribution of energy for the wire employed by me, but unfortunately this was not possible with the very unfavourable conditions of the Erlanger Institute-it is so exceptionally damp that it is not possible to set up rock-salt prisms \& c . for the purposes of an extended research. 
In our case

$$
a=0 \cdot 0_{2} 83 \text {. }
$$

If we make the slit so wide that when illuminated by homogeneous light of wave-length $\lambda$ it has a breadth in the spectrum corresponding to a difference of wave-length $\Delta$ at this place, and if we now illuminate it with white light, then every point at the same place receives rays between the wave-lengths $\lambda$ and $\lambda+\Delta$.

If the ordinate corresponding to the wave-length $\lambda$ in the energy-curve is $y$, and that corresponding to $\lambda+\Delta$ is $y_{1}$, then, since $\Delta$ is always small, the area included by the ordinates $y$ and $y_{1}$, the curve, and the axis of abscissa is

$$
\mathrm{F}=\frac{y+y_{1}}{2} \Delta,
$$

and the corresponding energy is

$$
\boldsymbol{a} \frac{y+y_{1}}{2} \Delta \text {. }
$$

The breadth $\Delta$ of the slit illuminated by the sodium flame in our experiments amounted to 0.22 of the distance between the sodium and lithium lines in the spectrum; the wavelength of the sodium line is 0.59 , of the lithium line 0.67 . Each point of the spectral image receives then rays between the wave-lengths $\lambda=0.59$ and $\lambda+\Delta=0.59+(0.67-0.59)$ $0 \cdot 22=0.6076$. Further, to the abscissæ 0.59 and 0.6076 correspond the ordinates $y=11.35$ and $y_{1}=13.33$; the abovementioned surface is therefore

$$
\mathrm{F}=\frac{11 \cdot 35+13 \cdot 33}{2} \times 0 \cdot 0176=\frac{24 \cdot 68}{2} \times 0 \cdot 0176 .
$$

But to this surface there corresponds a fraction $\xi$ of the total energy

$$
\xi=0 \cdot 0283 \times \frac{24 \cdot 68}{2} \times 0 \cdot 0176=0 \cdot 00180=\frac{1}{556} .
$$

Having thus determined the energy corresponding to this definite breadth of slit from measurements with our apparatus, we find for the sodium flame the whole, but for the platinum wire only the $\frac{1}{55_{6}}$ of the total radiated energy.

$\mathrm{A}^{\prime}$ and $\mathrm{A}$ are the measured brightnesses of the sodium flame and of the platinum wire, in reference to that of the amyl-acetate lamp; they are proportional to the squares of the cotangents of the readings on the photometer.

$$
\mathrm{A}^{\prime}=\text { const. } \operatorname{cotan}^{2} a^{\prime}, \quad \mathrm{A}=\text { const. } \operatorname{cotan}^{2} a,
$$

where the constants have the same value. 
Hence

$$
\frac{\mathrm{A}^{\prime}}{\mathrm{A}}=\frac{\operatorname{cotan}^{2 l} a^{\prime}}{\operatorname{cotan}^{2} a}
$$

therefore

$$
\mathrm{E}^{\prime}=0 \cdot 24 \frac{\operatorname{cotan}^{2} a^{\prime}}{\operatorname{cotan}^{2} a} \xi \mathrm{E} \text {. }
$$

In our case

so that

$$
a=36^{\circ} 30^{\prime}, \quad \mathrm{E}=4 \cdot 7, \text { and } \xi={ }_{55 \overline{6}},
$$

$$
\mathrm{E}^{\prime}=0.24 \frac{4 \cdot 7 \operatorname{cotan}^{2} a^{\prime}}{55 \operatorname{cotan}^{2} 36 \frac{1}{2}}=0.00203 \frac{\operatorname{cotan}^{2} a^{\prime}}{\operatorname{cotan}^{2} 36 \frac{1}{2}} \mathrm{~cm} \text {. g. sec. cal. }
$$

Coefficient of Total emission of $1 \mathrm{gr}$. sodium in absolute measure.

25. In order to test, in the first place, the dependence of the emission of light upon the quantity of sodium chloride contained in the unit volume, two solutions of sodium chloride were scattered into a flame in the mode described by Herr Ebert, exactly in the same way. They contained in 1 cub. centim. respectively

(a) $\mathrm{M}=0.0304 \mathrm{gr}$. sodium, (b) $\mathrm{M}_{1}=0.0132 \mathrm{gr}$. sodium. Their density was nearly unity.

A portion of the flame was placed opposite to the slit, which appeared uniform in the whole section. Its diameter is 2 cub. centim.

The readings $a^{\prime}$ on the photometer and the corresponding $\operatorname{cotan}^{2} a^{3}$ were for

Therefore, very nearly,

$$
\begin{array}{ll}
\text { (a) } a^{\prime}=31^{\circ}, & \operatorname{cotan}^{2} a^{\prime}=2 \cdot 770, \\
\text { (b) } a_{1}^{\prime}=42^{\circ}, & \operatorname{cotan}^{2} a_{1}^{\prime}=1 \cdot 233 .
\end{array}
$$

viz. :-

$$
\mathrm{M}: \mathrm{M}_{1}=\operatorname{cotan}^{2} a^{\prime}: \operatorname{cotan}^{2} a_{1}^{\prime} \text {, }
$$

The brightness therefore increases nearly proportionally to the quantity of salt present, which is also what Herr Gouy * has found.

For solutions of sodium carbonate, which contain in the unit volume the same quantities of sodium as the above sodium chloride solutions, the same brightnesses were found.

26. We will now calculats the quantity of sodium which in the first of these solutions yields the observed brightness, and the corresponding quantity of energy.

2100 cub. centim. of gas-mixture pass through the burner per minute + . The velocity at this point is, therefore,

* Ann. de Chem. et de Phys. [5] xviii. p. 5 (1879).

$\dagger$ Cf. also H. Ebert, Wied. Ann. xxxiv. p. 83 (1888). 


$$
\frac{2100}{3 \cdot 14 \times 1^{2}}=670 \text { centim. }
$$

i.e. in each minute a column of gas 670 centim. long passes in front of the slit. In 30 minutes $1.025 \mathrm{gr}$. would be scattered, therefore in 1 minute $0.034 \mathrm{gr}$. A column of 1 centim. height and 2 centim. diameter contains therefore

$$
\frac{0.034}{670}=5 \times 10^{-5} \mathrm{gr} .,
$$

and in 1 cub. centim.

$$
\frac{5 \times 10^{-5}}{3 \cdot 14}=1.59 \times 10^{-5} \mathrm{gr} \text {. fluid dust. }
$$

With the concentration chosen 1 cuh. centim. of the flame contains

$$
4.8 \times 10^{-7} \mathrm{gr} \text {. sodium. }
$$

Let us now calculate the quantity of sodium in a parallelopiped of unit height and breadth, therefore of the unit of radiating surface and of the thickness of the flame as depth, i.e. 2 cub. centim.; it contains in round numbers

$9 \cdot 6 \times 10^{-7} \mathrm{gr}$. sodium.

This quantity of $9.6 \times 10^{-7} \mathrm{gr}$. sodium therefore radiates the quantity of energy per second

$$
\mathbf{E}^{\prime}=0.00203 \frac{\text { contan }^{2} 31}{\operatorname{contan}^{2} 36 \frac{1}{2}}=0.00308 \mathrm{~cm} . \text { g. sec. calories. }
$$

The coefficient of total emission of sodium, i. e. the quantity of energy radiated by $1 \mathrm{gr}$. sodium in the two yellow lines of the Bunsen flame amounts to

$$
3210 \text { g.calories per second, }
$$

from which we obtain upon the assumption (no doubt not strictly correct) of equal brightness, $1600 \mathrm{gr}$. calories per second for each line.

An atom of sodium weighing $1.7 \times 10^{-21}$ gr. emits per second

$$
5 \cdot 5 \times 10^{-18} \mathrm{gr} \text {. calories. }
$$

27. We found before that $1 \mathrm{gr}$. platinum radiates on the whole $2 \cdot 2 \times 10^{4}$ gr. calories per second, now we find that with sodium for the two isolated spectrum lines the same value amounts to $3 \cdot 2 \times 10^{3}$, which is not so much less. It is as if the energy emitted, which with platinum is distributed throughout the entire spectrum, were with sodium concentrated in the two lines.

In the case of sodium we have besides the energy of the infra-red rays present according to the researches of Ed. 
Becquerel *, so that the coefficient of total emission of sodium for all rays together is greater than $3 \cdot 2 \times 10^{3}$.

True Coefficient of Emission of $1 \mathrm{gr}$. Sodium. Comparison with Platinum. Application of Kirchhoff's Law.

28. From the data for the coefficient of total emission for sodium, and the breadth of the sodium lines, we may obtain the true coefficient of emission for the unit breadth in the spectrum ( $c f$. p. 161) for $1 \mathrm{gr}$. sodium.

For this purpose we have only to divide the energy emitted by the breadth of a sodium line; this according to diffraction experiments (see below 32, p. 265) is $\frac{1}{4}$ of the distance between the centres of the two sodium lines-that is $0.15 \mu \mu$. The true coefficient of emission is then, if we take $1 \mu \mu$ as unit for the wave-length,

$$
\mathrm{S}_{\mathrm{N} \mathbf{2}}=\frac{1600}{0 \cdot 15}=10700 ;
$$

i. e. $1 \mathrm{gr}$. sodium in a region of the spectrum of the breadth $1 \mu \mu$ would emit per second $10700 \mathrm{~g}$. cal., or in round numbers $1.1 \times 10^{4}$ if the brightness throughout this region were alike.

29. We may further compare the true coefficient of emission of sodium vapour with that of solid platinum. We have seen above (p. 259) that since the ordinate of the curve of energy of platinum at the point which corresponds to the sodium line is $11 \cdot 35$, the energy eradiated within the region of the spectrum of breadth $1 \mu \mu$ by platinum is

$$
\xi \mathrm{E}=0 \cdot 0_{2} 83 \times 11.35 \times 0 \cdot 001 \times \mathrm{E}=9 \cdot 4 \times 10^{-3} \mathrm{E} \text {. }
$$

Thus 1 gr. platinum emits ( $c f$. p. 254) in this region

$$
9 \cdot 4 \times 10^{-5} \times 2 \cdot 2 \times 10^{4}=2 \cdot 1 \text { cal. }
$$

The ratio of the true coefficient of emission of sodium $S_{\mathrm{N} x}$ and platinum $\mathrm{S}_{\mathrm{Pt}}$ is therefore per gramme

$$
\frac{\mathrm{S}_{\mathrm{Na}}}{\mathrm{S}_{\mathrm{Pt}}}=\frac{1 \cdot 1 \times 10^{4}}{2 \cdot 1}=5 \times 10^{3} \text {. }
$$

The coefficient for sodium is thus much greater than that for platinum.

30. A film of platinum of 1 sq. centim. area and $10^{-5}$ centim. thickness, which contains $2 \times 10^{-4} \mathrm{gr}$. platinum is almost opaque.

According to Kirchhoff's law a film of sodium vapour which for equal area contains less substance in consequence of its greater coefficient of emission for its particular rays,

* Ed. Becquerel, Compt. Rend. xcix. p. 374 (1884). 
must also be opaque; i.e. a film which contains per square centimetre

$$
\frac{2 \times 10^{-4}}{5 \times 10^{3}}=4 \times 10^{-8} \text { gr. Na. }
$$

In the sodium flame examined by us there is present in a layer of 1 square centimetre $6.9 \times 10^{-7} \mathrm{gr}$., that is about twenty times as much as would be necessary to produce such opacity with the platinum.

If, therefore, Kirchhoff's law is to hold good here the flame must be absolutely opaque for the yellow rays. In fact such a flame shows reversal; i.e. in the centre of each of the yellow sodium lines a dark line appears when a ray of white light traverses it, nevertheless the absorption is by no means so great as one w'ould have expected according to Kirchhoff's law, since the dark line is confined to the centre. Hence it would seem that in the sodium flame luminescence phenomena appear together with with the usual luminous phenomena. In fact, highly complicated chemical processes occur in such a flame; further researches will show this more clearly.

\section{Direct Comparison of the Coefficients of Total and True Emission of Platinum and Sodium.}

31. In continuation of the foregoing, an experimental arrangement was employed, which will find frequent application in later investigations.

In one and the same flame sodium was distributed and a platinum wire was heated to luminosity, and the brightness of each was compared with the amyl-acetate lamp.

The apparatus slown in fig. 3 (P्Pl. III.) was employed. In the interior of an Ebert's* burner, B, a thin platinum-wire 0.26 millim. thick was fastened at $a$; it had thus the same thickness as the wire previously investigated, which was heated by the current; at its upper end it was attached to a small hook which was suspended to one arm of a lever, movable about the horizontal axis $e$, which was capable of being adjusted as to height. The lever was weighted on the other side by the weight $f$ in order to keep the wire stretched when hot.

Then water only was scattered in the flame, which was colourless, and the brightness of the platinum wire was measured ; then by a slight movement of the support the wire was put out of the field of view, the flame was fed with sodium solution, and its brightness measured again. 'These measurements showed that the ratio of brightness of the pla-

* Wied. Ann. xrxii. p. 345 (1887). 
tinum wire to that of the sodium flame was almost the same as in the previous experiments in which the wire was heated by a current. There is no object in giving the particular values here.

In exactly the same way experiments were made with solution of strontium chloride, which yields a spectrum of bands, solutions equivalent to the sodium chloride solution being scattered in the flame. The result obtained was that the total brightness is of the same order as that of the sodium flame, which also may be inferred from the strong coloration of the flame.

Here, therefore, nearly the same total energy is distributed over a series of bands.

32. We will now determine the ratio of the true coefficients of emission of two sources of light. For this purpose let us consider the following points:-

If we have a narrow spectral line whose boundaries are at $\lambda_{1}$ and $\lambda_{2}$, and if we examine this with a spectral-photometer of small dispersion, it appears in the spectrum of the same width as the slit. Let the dispersion be so chosen that the edges of the slit correspond to wave-lengths $\lambda$ and $\lambda+\Delta$ in the spectrum. If then, by means of a spectrophotometer, we compare the brightness of the spectrum-line $\lambda$ and of a continuous spectrum, then each point in the image of the slit, corresponding to the line-spectrum, receives rays between $\lambda_{1}$ and $\lambda_{2}$, and in the image of the slit corresponding to the continuous spectrum each point receives rays between $\lambda$ and $\lambda+\Delta$. If the intensities corresponding to the wavelength $\lambda$ are in the two cases $i^{\prime}$ and $i^{\prime \prime}$ respectively, then the total intensities $J_{1}$ and $J_{2}$ in the first and second image respectively are :-

$$
\mathrm{J}_{1}=\int_{\lambda_{1}}^{\lambda_{2}} i^{\prime} d \lambda \text { and } \mathrm{J}_{2}=\int_{\lambda}^{\lambda+\Delta} i^{\prime \prime} d \lambda .
$$

The quantities $i^{\prime}$ and $i^{\prime \prime}$ are proportional to the true coefficients of emission ; the ratio of the intensities is therefore

$$
\mathrm{V}=\int_{\lambda_{1}}^{\lambda_{2}} i^{\prime} d \lambda: \int_{\lambda}^{\lambda+\Delta} i^{\prime \prime} d \lambda
$$

In the simplest case we may assume that $i^{\prime}$ and $i^{\prime \prime}$ are constants, i.e. that the spectral lines between $\lambda_{1}$ and $\lambda_{2}$, as also the continuous spectrum between $\lambda$ and $\lambda+\Delta$, possess everywhere the same brightness, then

$$
\mathrm{V}=\frac{i^{\prime}}{i^{\prime \prime}} \cdot \frac{\lambda_{2}-\lambda_{1}}{\Delta}
$$

The observed ratio of intensities is therefore proportional to 
the ratio of the true coefficients of emission multiplied by $\left(\lambda_{2}-\lambda_{1}\right) / \Delta$.

We may determine $i^{\prime} / i^{\prime \prime}$ directly, if we observe with a spectrophotometer possessing so great a dispersion that the line also appears as a continuous band, for then the slit appears illuminated by homogeneous light of a width which is distinctly smaller than the spectral image of the line, it behaves therefore as a portion of a continuous spectrum; instead of $\lambda_{2}-\lambda_{1}$ we have $\Delta$, and that the more accurately the greater the dispersion, and the ratio of brightness measured in the apparatus is itself $i^{\prime} / i^{\prime \prime}$.

In order to determine the ratio $i^{\prime} / i^{\prime \prime}$ for a sodium flame and the amyl-acetate lamp, the following arrangement was made:The spectrum apparatus consisted of two telescopes belonging to Herr Ebert and a plane Rowland's grating. The collimator was provided with an arrangement for symmetrically narrowing down the slit, and had an aperture of 65 millim. and a focal length of 1 metre; the observing-telescope an aperture of $75 \mathrm{millim}$. and a focal length of 1 metre. The grating had a divided surface of $46 \times 36$ millim. and possessed very good definition. The slit was made very narrow. For the determination of the maximum of brightness a totallyreflecting prism was placed before one half of the slit; the beam of light from an arc lamp fell upon the uncovered half of the slit, after having traversed two adjustable Nicols. The light from the sodium flame or amyl-acetate lamp entered the apparatus through the totally-reflecting prism. First the sodium flame was brought before the prism, the apparatus adjusted for the greatly expanded sodium lines of the fourth spectrum by turning the grating, and the spectrum of the arc lamp weakened by turning one of the Nicols until it appeared of equal brightness with the sodium lines in that part of the spectrum.

Then the sodium flame was replaced by the amyl-acetate lamp, and adjusted for the yellow of the first diffractive spectrum. Since the light of the electric lamp was much brighter than that of the amyl-acetate lamp it was weakened by clouded glasses of known strength till the two spectra were of equal brightness. A direct comparison of brightness between the amyl-acetate lamp and the sodium flame in the fourth spectrum was not possible, because in the fourth spectrum the first was hardly visible. But since for all sources of white light the degree of weakening in passing from one spectrum to another is the same, and all continuous spectra are dispersed in the same degree, the ratio of brightness of amyl-acetate to arc-light must be the same in the 
spectra of different orders. This is also confirmed by a simple experiment. If an equality of brightness between the arc-light and the amyl-acetate light had been established in the first spectrum by the use of clouded glasses, this equality was also observed in the second and third spectra.

The comparison in brightness between the arc lamp and amyl-acetate lamp was made at a point a little distance from the yellow, on the side of the green, so that it might not be disturbed by sodinm contained in the arc. The results of experiment are as follows:-The breadth of a sodium line is $\frac{1}{4}$. of the distance between the two lines. The brightness diminishes from the centre rapidly, then more slowly, and at the edges more rapidly again. The measurements were made at about $\frac{1}{4}$ of the breadth from the edge after the brightness of the arc-light had been made equal to that of the sodium flame by rotation of the Nicol, it had then to be reduced to $\frac{1}{34}$ to be of the same brightness as the amyl-acetate lamp.

The brightness of our sodium lamp therefore for rays situated about $\frac{1}{4}$ of the breadth of a sodium line from its edge is 34 times greater than that of the amyl-acetate lamp at the corresponding point.

We may now apply the above results to the experiments on p. 261, where the brightness of the amyl-acetate lamp was compared with that of the sodium flame.

We will take $i^{\prime}$ to refer to the sodium flame, and $i^{\prime \prime}$ to the amyl-acetate lamp. In the sodium double lines the breadth of a single line is $\frac{1}{4}$ of the distance between the centres of the lines, so that the total breadth of the two together is $\frac{1}{2}$ this distance; the distance of the centres is known to be $0.0006 \mu$, consequently the quantity $\lambda_{2}-\lambda_{1}=0.0003 \mu$; further $V$ was found to be $2 \cdot 7$, since $\Delta=0.0176 \mu$ (p. 259) therefore

$$
\frac{i^{\prime}}{i^{\prime \prime}}=2.77 \times \frac{0.0176}{0.0003}=162 \text {. }
$$

The direct determination for a point about $\frac{1}{4}$ from the edge of the weakerline gave $\frac{i^{\prime}}{i^{\prime \prime}}=34$. It follows from the difference of these numbers, which agree as to order of magnitude, that the above assumption as to the distribution of brightness is not strictly correct, but that the brightness of a sodium line must increase rapidly in the centre, which is also confirmed by the appearance to the eye. This further indicates the possibility that in spite of the breadth of the spectral lines, interferences may occur with differences of path up to more than 100,000 wave-lengths, such as have been observed by several experimenters.

[To be continued.] 